\title{
Mediación en una Comunidad Intercultural
}

\section{Mediation at an Intercultural Community}

\author{
Inmaculada Urruela Arnal \\ Equipos Técnicos de Asesoramiento en Justicia de Menores, España
}

\author{
Iñaki Bolaños Cartujo \\ Universidad Complutense de Madrid, España
}

\begin{abstract}
Resumen. En este trabajo se pretende reflexionar sobre las formas que ha adquirido en nuestro país la aplicación de la mediación en contextos comunitarios, dando cuenta de la especificidad que adquiere cuando el espacio de relación social es un lugar donde la diversidad cultural es una realidad. Se analizan las definiciones, conceptos clave, objetivos y fundamentos metodológicos y teóricos de su práctica. Para ello, se utilizan documentos originales, recogiendo textualmente las definiciones y exposiciones teóricas alrededor del concepto mediación intercultural i/o comunitaria. El objetivo de utilizar las expresiones literales es poder analizar posteriormente las diferentes categorías recogidas en los redactados. Hemos entendido que el análisis de contenidos, evitando el análisis de los semánticos empleados, perdería parte de la intención de los autores originales. La elección de las palabras no es casual. Su análisis pormenorizado nos lleva a contrastar prioridades y principios y nos acerca al objetivo del trabajo que es contrastar las diferencias y sobre todo identificar las similitudes.

Palabras clave: mediación intercultural, mediación comunitaria, diversidad, convivencia, ciudadanía.
\end{abstract}

\begin{abstract}
This work tries to think about the forms that the application of the mediation has acquired in our country in community contexts, realizing of the specificity that he acquires when the space of social relation is a place where the cultural diversity is a reality. There are analyzed the definitions, key, objective concepts and methodological and theoretical foundations of his practice. For it original documents are in use, gathering textually the definitions and theoretical exhibitions about the concept intercultural mediation and community mediation. The aim to use the literal expressions is to be able to analyze later the different categories gathered in the written ones. We have understood that the analysis of contents, avoiding the analysis of the semantic ones, would lose part of the intention of the original authors. The choice of the words is not chance. His detailed analysis leads us to confirming priorities and beginning and brings us over to the aim of the work that is to confirm the differences and especially to identify the similarities.

Keywords: intercultural mediation, community mediation, diversity, living together, citizenship.
\end{abstract}

El arte de la paz no es fácil. Es una lucha hasta el fin, la matanza de los malos deseos y de la falsedad interior. En algunas ocasiones la voz de la paz resuena como un trueno, sacudiendo a los seres humanos y sacándolos de su letargo.

(Ueshiba, 2003)

La correspondencia sobre este artículo debe enviarse a Iñaki Bolaños Cartujo, e-mail: jibolanos@psi.ucm.es

\section{Introducción}

La experiencia de la diferenciación con el otro puede ser el origen de innumerables conflictos en cualquier ámbito en que se desarrollen. Desde el nacimiento, la construcción de la identidad requiere de la evidencia de una otredad diferencial. No somos si no somos diferentes. Entonces, podemos pensar que la diversidad nos da la oportunidad de ser 
nosotros. Sin embargo, la experiencia de la pluralidad fuera nos pone a prueba y los lugares de encuentro que son origen de satisfacción, son también generadores de intensos conflictos. La oportunidad del conflicto, por tanto, forma parte de nuestra realidad como sujetos sociales.

Las sociedades han desarrollado formas de subsistir pese al otro, intentando preservar lo conocido como universal y lo ajeno como amenaza, asimilando o anulando la diferencia. Por su parte, la mediación vinculada en su origen a la resolución menos dolorosa y más responsable de los conflictos en el ámbito familiar, se ha constituido en un instrumento formal válido también para tejer nexos de diálogo y pacificación entre individuos, grupos e instituciones, dando respuesta al deseo de superar la diferencia mediante el diálogo.

En este trabajo se incorporan algunos apuntes de mediación comunitaria y mediación intercultural. Como fuentes de la mediación en nuestro país hemos seleccionado dos experiencias claves por resultar promotoras, cada una en su ámbito geográfico, y por el desarrollo que han potenciado de esta practica profesional. En Madrid, el Servicio de Mediación Social Intercultural SEMSI (19972010), nace de la colaboración entre el Ayuntamiento de Madrid y la Universidad Autónoma y permaneció integrado durante su trayectoria en las actuaciones del Programa de Atención a la Inmigración e Interculturalidad con una estrecha colaboración con los equipos técnicos de los servicios municipales. La experiencia de trabajo del SEMSI se recoge sistematizada en el libro "El servicio de mediación social intercultural SEMSI 1997-2002" editado por el Área de Servicios Sociales del Ayuntamiento de Madrid (2002) que hemos utilizado por su claridad expositiva. En Catalunya, la publicación del Libro Blanco de la mediación (Casanovas, Magre y Lauroba, 2010), nos ha permitido contar con una información detallada de la evolución de la práctica de la mediación comunitaria y /o intercultural, haciendo referencia a su trayectoria, diversidad según su aplicación geográfica y dependencia.

El I Congreso Internacional sobre Mediación Intercultural desarrollado en Madrid en octubre de 2002 y promovido por el Programa Migración y
Multiculturalidad de la UAM y el Ayuntamiento de Madrid, resultó un lugar de encuentro y reflexión, poniendo en común la experiencia en diferentes ámbitos de aplicación, así como aspectos del proceso, formación de profesionales y rol de las personas mediadoras.

En la evolución de la mediación intercultural y comunitaria en España fue definitivo el I Encuentro de Mediadoras y Mediadores Interculturales en Valencia en el año 2007. Fue organizado por el Grupo Triángulo, que en ese momento estaba formado por entidades de Andalucía, Catalunya y Madrid: Etnic (Granada), Federación Andalucía Acoge, Sevilla Acoge (Sevilla), Fundación secretariado gitano (Granada), ACISI (Barcelona), Desenvolupament Comunitari (Barcelona), KAB (Barcelona) y el Programa Migración y multiculturalidad de la Universidad Autónoma de Madrid. Se realizaron tres jornadas intensas de trabajo pormenorizado sentando las bases de una práctica profesional que, con trayectorias distintas, se había puesto en marcha años atrás en nuestro país desde diferentes entidades. El encuentro estaba justificado por la necesidad de conseguir un marco común de intervención, una identidad compartida surgida de la reflexión sobre la experiencia de los diferentes actores y que superase la ausencia de una normativa previa que consensuara tanto la formación como la práctica. La experiencia se llevó a cabo con la metodología de talleres participativos con el objetivo general de "avanzar en el reconocimiento oficial de la figura mediadora intercultural, desde un marco común, como vía para garantizar la calidad, la competencia y la colaboración en el desarrollo de esta profesión" (Grupo Triángulo, 2007). El trabajo fue más allá de la definición y los objetivos y permitió consolidar un nuevo escenario profesional. Se evaluaron y debatieron a través de grupos de trabajo aspectos como la formación, relación entre personas mediadoras, conocimiento y reconocimiento de la mediación intercultural, modelo de la mediación intercultural y perfil de la figura mediadora, así como sobre la profesión, práctica y condiciones laborales.

El objetivo final es evidenciar similitudes más que diferencias. Nos preguntamos si la mediación comunitaria es intercultural y si es comunitaria la 
mediación intercultural y, más allá todavía, si ambas encajan realmente en el concepto de mediación. En este sentido queremos reconocer las aportaciones de Carlos Giménez, director del Programa Migración y multiculturalidad de la Universidad Autónoma de Madrid. Si hay alguien que ha sabido avanzar en las respuestas a estas preguntas, es él. Es verdad que la práctica de la mediación intercultural no siempre parece responder a los parámetros más ortodoxos de la mediación. Entender ésta y entender el concepto interculturalidad como dos elementos que definen una auténtica manera de pensar y hacer la mediación, es algo que debemos al esfuerzo, no siempre suficientemente reconocido, de nuestro maestro.

\section{Mediación comunitaria}

\subsection{Definición}

"Un recurso humano y un instrumento cívico mediante el cual los integrantes de una sociedad pueden tramitar sus diferencias y/o gestionar los conflictos que se les presentan en el ámbito privado y/o público, así como también participar en la construcción de la sociedad que integran” (Nató, Rodríguez y Carbajal, 2005).

\section{2. Ámbitos de actuación}

Conflictos que se pueden abordar en el ámbito social comunitario:

- Conflictos en la comunidad. Tanto en el interior como hacia el exterior de dicha comunidad.

- Conflictos públicos. Pueden referirse a dos o más actores, sean grupos, organizaciones, asociaciones vecinales, empresas, etc. Son en cualquiera de los casos conflictos de interés público.

- Conflictos interculturales. "Evolución desde el multiculturalismo hacia la interculturalidad, en busca de una integración basada en el respeto y en el reconocimiento reciproco." (Nató y col., 2005).

\section{Primer Encuentro de Mediadoras y Mediadores Interculturales. Valencia 2007. Grupo Triángulo}

\subsection{Definición}

Entienden la mediación intercultural "como un proceso que contribuye a mejorar la comunicación, la relación, y la integración intercultural entre personas o grupos presentes en un territorio y pertenecientes a una o varias culturas y con códigos culturales diferentes" (Grupo Triángulo, 2007). Esta definición aporta la idea de proceso, compartida por la mediación en general. Se amplía así la definición ofrecida cinco años antes por Desenvolupament Comunitari como un "recurso profesionalizado que pretende contribuir a una mejor comunicación, relación e integración entre personas o grupos presentes en un territorio, y pertenecientes a una o varias culturas" (Bermúdez, Castro y García., 2002).

\section{2. Ámbito}

Todos los ámbitos de la mediación en general, en donde existen diferencias culturales significativas en el conflicto.

\subsection{Funciones de la persona mediadora}

- Facilitar la comunicación.

- Intervenir en la prevención, resolución y transformación de conflictos y tensiones.

- Asesorar a los agentes sociales en temas de interculturalidad.

- Promover el acceso a los servicios y recursos.

- Construir ciudadanía.

- Favorecer la participación.

\subsection{Tipologías (acciones)}

- Preventiva. Facilitar el acercamiento, la comunicación y la comprensión entre personas /grupos con diferentes códigos culturales. 
- Rehabilitadora. Regulación y resolución de conflictos entre individuos o grupos con códigos culturales distintos.

- Transformadora. Proceso creador. Superar las normas para alcanzar nuevas normas y modos de relación.

\section{Mediación en Madrid: SEMSI} (Años 1997-2010)

El Servicio de Mediación Social Intercultural (SEMSI) fue creado mediante un convenio entre el Área de Servicios Sociales del Ayuntamiento de Madrid y el programa Migración y Multiculturalidad de la Universidad Autónoma de Madrid. De forma previa a establecer este convenio de colaboración, el programa tenía entre sus objetivos tanto el estudio del fenómeno migratorio como la formación de especialistas en contextos de inmigración y multiculturalidad a través de la gestión de la Escuela de Mediadores Sociales para la Inmigración (EMSI) de la Comunidad de Madrid.

\subsection{Definición}

Se parte de la definición de Mediación Intercultural desarrollada por Carlos Giménez (1997), entendida como una modalidad de mediación, en cuanto a intervención de terceras partes, que tiene lugar en contextos y situaciones de "multiculturalidad significativa", que se puede dar entre actores sociales o entre instituciones etnoculturalmente diferenciadas. Textualmente Giménez define una situación de multiculturalidad significativa como "aquellas situaciones sociales en las que están involucrados individuos, grupos o instituciones diferenciadas culturalmente entre sí y concebimos esa diferenciación cultural en el sentido más amplio, incluyendo los contrastes por etnicidad, raza, religión lengua y nacionalidad" (Giménez, 1997).

Según la perspectiva de este autor, por tanto, la mediación intercultural es una modalidad de mediación social diferenciada de otras formas de acción social que pueden estar representadas en la práctica comunitaria.

\subsection{Objetivos}

Continúa la definición refiriendo la orientación que debe tener esta práctica:

- Consecución del reconocimiento del otro.

- Acercamiento de las partes.

- Comunicación y comprensión mutua.

- Aprendizaje y desarrollo de la convivencia.

- Regulación de conflictos.

- Adecuación institucional.

\subsection{Perfil de la persona mediadora}

- Formación en mediación, interculturalidad e inmigración.

- Especialista en mediación comunitaria en contextos de multiculturalidad.

- Puente entre las personas inmigrantes y las instituciones, la población extranjera y la autóctona.

- Pertenencia a un equipo diverso e intercultural.

- Preferentemente extranjeras, pero no únicamente.

\subsection{Planteamiento teórico}

- Concepción de la mediación intercultural como una de las modalidades de la mediación general. Énfasis en los principios de la mediación.

- Perspectiva intercultural como complementaria del multiculturalismo y superadora de sus límites.

- Se media en "situaciones sociales de multiculturalidad significativa". Aplicación de un planteamiento multifactorial, que distingue e interacciona los factores personales, situacionales y culturales (Giménez, 1997, 2002).

\subsection{Niveles de intervención}

3.5.1 Mediación interpersonal. Mediación en atención de casos (particulares o representantes de instituciones). 


\section{Objetivos}

- Apoyo a profesionales de los Servicios Sociales en el desempeño de su labor.

- Puente entre personas usuarias y profesionales de servicios sociales, facilitando el acceso a los recursos.

- Facilitar instrumentos a la población de origen extranjero para desenvolverse en la sociedad de acogida.

\subsection{1. Ámbito comunitario}

- Conocimiento de la realidad.

- Identificación de problemáticas.

- Adecuación de recursos.

\section{Objetivos}

El general es promover la convivencia intercultural definida como vivir cotidiano entre personas que presentan distintos bagajes culturales, en el cual interaccionan entre sí dando lugar a relaciones sociales e intercambios, acercamiento, etc. que desemboca en la construcción de una nueva cultura compartida sin que esto implique la eliminación de las identidades de origen, aunque si su posible transformación y enriquecimiento (SEMSI, 2002).

Pero este objetivo general, se desarrolla por otros dos grandes objetivos:

- Contribuir a que la convivencia intercultural sea posible con los siguientes fines:

- El desarrollo comunitario.

- La prevención y gestión del conflicto.

- La construcción de una sociedad intercultural.

- Fomentar la participación ciudadana.

- Participación individual.

- Participación colectiva.

Los dos niveles se coordinan para cumplir los objetivos de prevención, resolución de conflictos y promoción de la convivencia.

\subsection{Formas de mediación intercultural}

Según los contextos de aplicación:

- Ámbito socio-jurídico.

- Ámbito laboral.

- Ámbito institucional.

- Ámbito educativo.

- Ámbito sanitario.

- Ámbito familiar.

- Ámbito vecinal.

Según los actores involucrados:

Se incluyen las relaciones entre individuos, grupos y comunidades en todas las formas posibles de relación entre ellos.

\section{Mediacion en Catalunya}

El libro blanco de la mediación en Catalunya (2010), al abordar el núcleo de la mediación comunitaria empieza por ubicarlo en el ámbito de la convivencia social, asociando en su práctica tres adjetivos claves: ciudadana, comunitaria e intercultural, subrayando que aunque son términos distintos tienen un mismo referente. No obstante, establece una diferencia clara entre la medición comunitaria y la mediación intercultural y ésta es la referida a las funciones de traducción e interpretación entre culturas distintas que junto a las prácticas comunes de resolución de conflictos, ejerce la mediación intercultural y que no tiene necesariamente la comunitaria.

\subsection{Modelo de mediación comunitaria del Ayun- tamiento de Barcelona}

Se divide en mediación comunitaria y mediación intercultural comunitaria

\subsubsection{Mediación comunitaria}

\subsubsection{Definición de mediación comunitaria}

Según el modelo del Ayuntamiento de Barcelona, Servei de Mediaciò i/o Gestiò de conflictes de la 
convivencia a Barcelona, recogido en el Libro blanco de la mediación en Catalunya, se trata de un "procedimiento de resolución de conflictos para aquellas problemáticas y para aquellos sujetos que cumplen con los requisitos y se adaptan a sus exigencias" (Casanovas, P. et al., 2010).

4.1.1.2. Objeto. Conflictos de convivencia dentro del ámbito privado. Demanda entre particulares

4.1.1.3. Finalidad. Mediación como estrategia de gestión de conflictos a través del dialogo y la corresponsabilización

4.1.1.4. Acceso. Recurso de segundo nivel. Se llega previa derivación del técnico de derivación o convivencia que selecciona el conflicto

\subsubsection{Tipología de conflictos}

- Conflictos "mediables" (p. e. convivencia vecinal).

- Conflictos "no mediables":

a. Delitos o hechos que incumplen la normativa municipal.

b. Delitos contra derechos fundamentales del individuo.

c. Si existe trastorno que imposibilite el razonamiento en una de las partes.

\subsubsection{Mediación intercultural comunitaria}

\subsubsection{Definición}

"El Servei de Mediació Intercultural está orientado al apoyo de personas, familias y profesionales, así como al fomento de relaciones vecinales y comunitarias de convivencia en contextos de diversidad cultural" (Casanovas, P. et al., 2010).

\subsubsection{Concepto de interculturalidad}

Comportamiento basado en la empatía y el descubrimiento mutuo.

\subsubsection{Objetivos de la mediación intercultural}

"Proveer recursos a los profesionales municipales y a la ciudadanía, tanto personas autóctonas como inmigradas, para facilitarles la comunicación, el dialogo y la convivencia intercultural, previniendo o resolviendo aquellos elementos de conflicto que se pueden dar en las relaciones ciudadanas" (Casanovas, P. et al., 2010).

Específicamente recoge los siguientes objetivos generales:

- Facilitar la acogida de la población inmigrada desde los servicios municipales.

- Posibilitar procesos de adecuación de la atención social a las necesidades y particularidades de la población recién llegada.

- Promover el dialogo entre personas y grupos de origen sociocultural diverso. Mediar en los conflictos derivados de la integración.

\subsubsection{4. Ámbito de actuación}

- Individual. Traducción e interpretación como servicio de apoyo los servicios municipales.

- Conflicto multiparte. Intervención en proyectos de dinamización comunitaria, con incidencia en el espacio público. Ámbitos comunitario, grupal y vecinal.

\subsection{Modelo de Mediación ciudadana}

Area de Igualtat i ciutadania de la Diputaciò de Barcelona

\subsubsection{Definición}

Mediación ciudadana. Complementaria de la mediación comunitaria y mediación intercultural.

\subsubsection{Marco teórico}

Políticas de ciudadanía. Papel activo de los ciudadanos en la resolución de sus propios conflictos. Valores: autonomía, corresponsabilidad, reconocimiento y respeto de la diversidad, participación de la ciudadanía. Liderazgo municipal. Autonomía local. La administración local es la responsable de de garantizar la convivencia y cohesión social. 


\subsubsection{Objetivo}

Prevención. Reducir la conflictividad social, fomentando la cultura de la paz. Concepto clave "ciudadanía residente". Concepto de ciudadanía basado en la residencia efectiva, reconocida por el empadronamiento.

\subsection{4. Ámbito de actuación}

Según conflictos atendidos.

- Derivado de la convivencia en las comunidades de vecinos.

- Derivados del uso y el empleo de los espacios públicos.
- Conflictos relativos a civismo.

- Derivados del ejercicio de actividades económicas.

- Conflictos en ámbito escolar.

- Conflictos en el ámbito de la convivencia familiar.

- Conflictos entre diferentes entidades o entre los miembros de una misma entidad.

\subsubsection{Funciones de la persona mediadora}

- Procesos de mediación en resolución de conflictos.

- Difusión de la cultura de paz, sensibilización.

- Prevención de conflictos.

RELACIÓN ENTRE LOS CONTENIDOS

\begin{tabular}{|c|c|c|c|c|}
\hline & $\begin{array}{l}\text { Mediación intercultural } \\
\text { (SEMSI) }\end{array}$ & $\begin{array}{l}\text { Mediación intercultural } \\
\text { comunitaria (SMMI) }\end{array}$ & $\begin{array}{l}\text { Mediación comunitaria } \\
\text { Barcelona }\end{array}$ & Mediación ciudadana \\
\hline DEFINICIÓN & $\begin{array}{l}\text { Modalidad de mediación } \\
\text { social }\end{array}$ & $\begin{array}{l}\text { Modalidad de mediación } \\
\text { comunitaria }\end{array}$ & $\begin{array}{l}\text { Modalidad de mediación } \\
\text { comunitaria genérica }\end{array}$ & $\begin{array}{l}\text { Complementa comunitaria } \\
\text { e intercultural }\end{array}$ \\
\hline ESPECIFICIDAD & $\begin{array}{l}\text { Contextos de multicultura- } \\
\text { lidad significativa }\end{array}$ & $\begin{array}{l}\text { Contextos de diversidad } \\
\text { cultural }\end{array}$ & $\begin{array}{l}\text { Convivencia ámbito priva- } \\
\text { do }\end{array}$ & $\begin{array}{l}\text { Corresponsabilidad ciuda- } \\
\text { dana }\end{array}$ \\
\hline ÁMBITO & $\begin{array}{l}\text { Relaciones entre indivi- } \\
\text { duos, grupos y comunida- } \\
\text { des }\end{array}$ & $\begin{array}{l}\text { Personas, familias profe- } \\
\text { sionales }\end{array}$ & $\begin{array}{l}\text { Demanda entre particula- } \\
\text { res. Previa derivacion }\end{array}$ & Ciudadanía residente \\
\hline OBJETIVO & $\begin{array}{l}\text { Promoción de la conviven- } \\
\text { cia }\end{array}$ & $\begin{array}{l}\text { Apoyo y fomento de rela- } \\
\text { ciones de convivencia en } \\
\text { diversidad cultural }\end{array}$ & $\begin{array}{l}\text { Gestión de conflictos } \\
\text { corresponsabilizacion }\end{array}$ & $\begin{array}{l}\text { Reducir la conflictividad } \\
\text { social } \\
\text { Fomentar la cultura de paz }\end{array}$ \\
\hline RECURSOS & $\begin{array}{l}\text { Reconocimiento } \\
\text { Comunicación } \\
\text { Convivencia }\end{array}$ & $\begin{array}{l}\text { Comunicación } \\
\text { Diálogo } \\
\text { Convivencia }\end{array}$ & Diálogo & \\
\hline CONFLICTO & $\begin{array}{l}\text { Prevención } \\
\text { Resolución }\end{array}$ & $\begin{array}{l}\text { Previniendo } \\
\text { Resolviendo }\end{array}$ & Gestión & $\begin{array}{l}\text { Prevención } \\
\text { Reducción }\end{array}$ \\
\hline ACCIONES & $\begin{array}{l}\text { Apoyo a profesionales } \\
\text { Prevención y gestión con- } \\
\text { flicto } \\
\text { Desarrollo comunitario } \\
\text { Participación ciudadana }\end{array}$ & $\begin{array}{l}\text { Acogida institucional } \\
\text { Diálogo } \\
\text { Mediar en conflictos } \\
\text { Traducción e interpretación } \\
\text { Dinamización comunitaria }\end{array}$ & $\begin{array}{l}\text { Resolución de conflictos } \\
\text { mediables } \\
\text { En ámbito vecinal }\end{array}$ & $\begin{array}{l}\text { Fomentar cultura de paz } \\
\text { Prevención de conflictos }\end{array}$ \\
\hline $\begin{array}{l}\text { CONTEXTO de } \\
\text { INTERVENCIÓN }\end{array}$ & $\begin{array}{l}\text { Sociojurídico } \\
\text { Laboral } \\
\text { Institucional } \\
\text { Educativo } \\
\text { Sanitario } \\
\text { Familiar } \\
\text { Vecinal }\end{array}$ & $\begin{array}{l}\text { Comunitario } \\
\text { Grupal } \\
\text { Vecinal } \\
\text { Individual. }\end{array}$ & $\begin{array}{l}\text { Convivencia vecinal } \\
\text { Recurso de segundo nivel. }\end{array}$ & $\begin{array}{l}\text { Espacios públicos } \\
\text { Escolar } \\
\text { Convivencia. Familiar } \\
\text { Civismo } \\
\text { Entidades }\end{array}$ \\
\hline
\end{tabular}




\section{Conclusiones}

- La mediación intercultural puede vincularse en su origen a la necesidad de crear un puente en el espacio que existe entre los departamentos o servicios de salud, educación y servicios sociales y las comunidades inmigrantes, como respuesta de la intervención social a los cambios poblacionales con motivo de los movimientos migratorios.

- La mediación intercultural incluye en su concepción la perspectiva lingüística como un espacio posible de intermediación.

- Comparte con otras formas de mediación la evolución que ha tenido la idea de resolución del conflicto de origen hacia la de gestión o trasformación. En este ámbito, superar el conflicto como foco de intervención es dirigir la atención hacia la convivencia. En todas las definiciones aparece el enfoque hacia el ámbito de la convivencia.

- La perspectiva de intervención intercultural de la mediación es transversal en el sentido de actuar en diferentes ámbitos de las relaciones sociales e interpersonales.

- La mediación intercultural incluye la perspectiva estructural de cambio social a través de la transformación de las relaciones entre los ciudadanos.

- Mediación social intercultural y mediación comunitaria intercultural son denominaciones que con diferente origen vinculan el nivel sociocomunitario con la gestión de conflictos en situaciones de interculturalidad relacional.

La mediación comunitaria/social es intercultural de facto si reconoce la pluralidad en el origen de la ciudadanía a la que se dirige su actuación. Las diferentes denominaciones responden a dependencias estructurales o institucionales. Así podemos encontrar una mediación denominada comunitaria en origen, que se diversifica en intercultural cuando el objeto de intervención esta señalado por una significativa distancia cultural y podemos encontrar una mediación denominada intercultural en su origen que se torna comunitaria si la diferenciación cultural que le de sentido de existir afecta a la totalidad de la comunidad, extendiendo entonces su actuación a este ámbito.

Y en definitiva, cualquiera que sea la denominación que adquiera, comparte con la generalidad de la práctica mediadora la certeza de que es posible un sistema de organización social que otorgue a los sujetos el protagonismo en su vida, reconociendo la madurez y responsabilidad que les corresponde. La función de la Mediación como promotora de Cultura de Paz, no es una alternativa, es la respuesta evolutiva del ser social que, insatisfecho de encontrarse con el otro por la vía de la confrontación y la extinción mutua, no ve más solución para sobrevivir que reconocer al otro y dialogar en la diferencia. La experiencia satisfactoria de esta búsqueda del otro se extiende, inevitablemente, a todos los espacios de relación.

\section{Referencias}

Bermúdez, K., Castro, R. y García, H. (2002). Mediación intercultural: Una propuesta para la formación. Madrid, Popular.

Casanovas, P., Magre, J. y Lauroba, M. E. (2010). Llibre blanc de la mediació a Catalunya. Barcelona, Departament de Justicia de la Generalitat de Cayalunya. Disponible en http://www.llibreblancmediacio.com

Giménez, C. (1997). La naturaleza de la mediación intercultural. Revista Migraciones, 2, 125-159.

Giménez, C. (2002). Planteamiento multifactorial para la mediación e intervención en contextos multiculturales: una propuesta metodológica de superación del culturalismo; en F. J. García Castaño y C. Muriel López (eds.), La inmigración en España: contextos y alternativas. Volumen II, (pp. 617644). Actas del III Congreso sobre la Inmigración en España (ponencias). Granada: Laboratorio de Estudios Interculturales.

Grupo Triángulo (2007). Documento base del Primer Encuentro de mediadoras y mediadores interculturales, Valencia: Autor.

Nató, A., Rodríguez, G. y Carbajal, L. (2005). Mediación Comunitaria. Conflictos en el escenario social urbano. Hermosillo, Sonora, México: Centro internacional de estudios sobre democracia y paz social.

SEMSI. (2002). El Servicio de Mediación Social Intercultural SEMSI. Madrid: Área de servicios sociales del Ayuntamiento de Madrid.

Ueshiba, M. (2003). El arte de la paz. Buenos Aires: Troquel.

Manuscrito recibido: 08/02/2012

Revisión recibida: 03/05/2012

Aceptado: 04/05/2012 